\title{
A Blockchain and FOT (Fog of Things) based system and method for predicting an Infectious disease transmitted by a virulent respiratory virus
}

\section{Dr. Naveen Tewari ( $\square$ navtewari@gmail.com )}

Graphic Era Hill University, Bhimtal, Uttarakhand, India

\section{Dr. Rajeev Kumar}

TMU, Mooradabad, UP, India

\section{Method Article}

Keywords: Blockchain, loT, sensors, FoT, Cloud, Virus, Disease

Posted Date: October 30th, 2020

DOl: https://doi.org/10.21203/rs.3.rs-100784/v1

License: (c) (1) This work is licensed under a Creative Commons Attribution 4.0 International License.

Read Full License 


\title{
A Blockchain and FOT (Fog of Things) based system and method for predicting an Infectious disease transmitted by a virulent respiratory virus
}

\author{
Dr. Naveen Tewari ${ }^{1}$, Dr. Rajeev Kumar ${ }^{2}$ \\ ${ }^{1}$ School of Computing, Graphic Era Hill University, Bhimtal, navtewari@gmail.com \\ ${ }^{2}$ College of Computing Sciences and Information Technology, Teerthankar Mahaveer \\ University, Moradabad, rajeev2009mca@gmail.com
}

\begin{abstract}
The present research is generally related to a system and method for predicting an infectious disease such as COVID-19 transmitted by a virulent respiratory virus. Disclosed are a system and method for predicting an infectious disease transmitted by a virulent respiratory virus. The system includes a plurality of Internet of Things (IoT) sensors, a plurality of fog node devices, and a plurality of computing devices in cloud data centers. The IoT sensors are configured to be coupled to a plurality of human beings to generate a health dataset. The fog node devices are associated with a fog layer to receive the health dataset from the IoT sensors to process and to store the health dataset over a blockchain network. The fog node devices process the health dataset at the fog layer by performing a fog computing. The computing devices and cloud data centers receive the processed health dataset from the of fog node devices over the blockchain network. This research is also filed for patent in Indian Patent Office with application number 202011021969. [5]
\end{abstract}

Keywords: Blockchain, IoT, sensors, FoT, Cloud, Virus, Disease

\section{Introduction}

\subsection{Background}

Typically, human migration and the spread of infectious diseases are interrelated. The emergence of COVID-19 and its subsequent dissemination to various countries provided irrefutable evidence that international and even intercontinental borders are highly permeable to the spread of infectious diseases. Whether originating from breaches at laboratories harboring dangerous infectious pathogens, 
bioterrorism, or natural causes, outbreaks of emerging diseases with pandemic potential constitute a vital threat to the health and economic security of the entire international community.

\subsection{Problem}

The health care services throughout the world are failing to contain and prevent the spread of COVID-19 (Corona virus) However, the existing systems and methods are inefficient and ineffective in predicting an infectious disease transmitted by a virulent respiratory virus. Therefore there is a need for an automated, Blockchain, and FOT (Fog of Things) based intelligent system and method to predict viral spread diseases like COVID-19, consequently helping in stopping spreading.

Thus, in view of the above, there is a long-felt need in the industry to address the aforementioned deficiencies and inadequacies. Further limitations and disadvantages of conventional and traditional approaches will become apparent to one of skill in the art through comparison of described systems with some aspects of the present disclosure, as set forth in the remainder of the present application and with reference to the drawings.

\subsection{Proposed Solution}

For the above problem, we hereby propose a present system and method provide a virus epidemic prediction system (VEPS). Further, the present system utilizes a blockchain network and FOT (Fog of Things) based intelligent system predicting viral spread diseases like COVID-19, consequently helping in stopping spreading.

This research paper also provides the detailed workflow of VEPS and proposes a solution to the problem. The present system and method transfer the health datasets via IoT to the local fog based system via a secured blockchain network. The fog computing (Fog-of-Thing) is used to perform processing of gathered data, before sending it to Cloud Datacenters. The fog system automatically sends alert to a mobile application or a computer application accessible to all the government authorities, and health monitoring agencies. This communication is also secured with the blockchain network. 


\section{Related Work}

\subsection{Background Research}

A publication titled "An intelligent and secure system for predicting and preventing Zika virus outbreak using Fog computing" filed by Sanjay Sareen et al. talks about a fuzzy k-nearest neighbor is used to diagnose the possibly infected users, and Google map web service is used to provide the geographic positioning system (GPS)-based risk assessment to prevent the outbreak. It is used to represent each Zika virus (ZikaV)-infected user, mosquito-dense sites, and breeding sites on the Google map that help the government healthcare authorities to control such riskprone areas effectively and efficiently. The proposed system is deployed on Amazon EC2 cloud to evaluate its performance and accuracy using data set for 2 million users. The system provides high accuracy of $94.5 \%$ for the initial diagnosis of different users according to their symptoms and appropriate GPS-based risk assessment. [4]

A PCT application WO2004092360A2 filed by Chien David et al. discloses "an outbreak of a virulent respiratory virus, now known as Severe Acute Respiratory Syndrome (SARS), was identified in Hong Kong, China and a growing number of countries around the world in 2003. The research relates to nucleic acids and proteins from the SARS corona virus. These nucleic acids and proteins can be used in the preparation and manufacture of vaccine formulations, diagnostic reagents, kits, etc. The research also provides methods for treating SARS by administering small-molecule antiviral compounds, as well as methods of identifying potent small molecules for the treatment of SARS." [19]

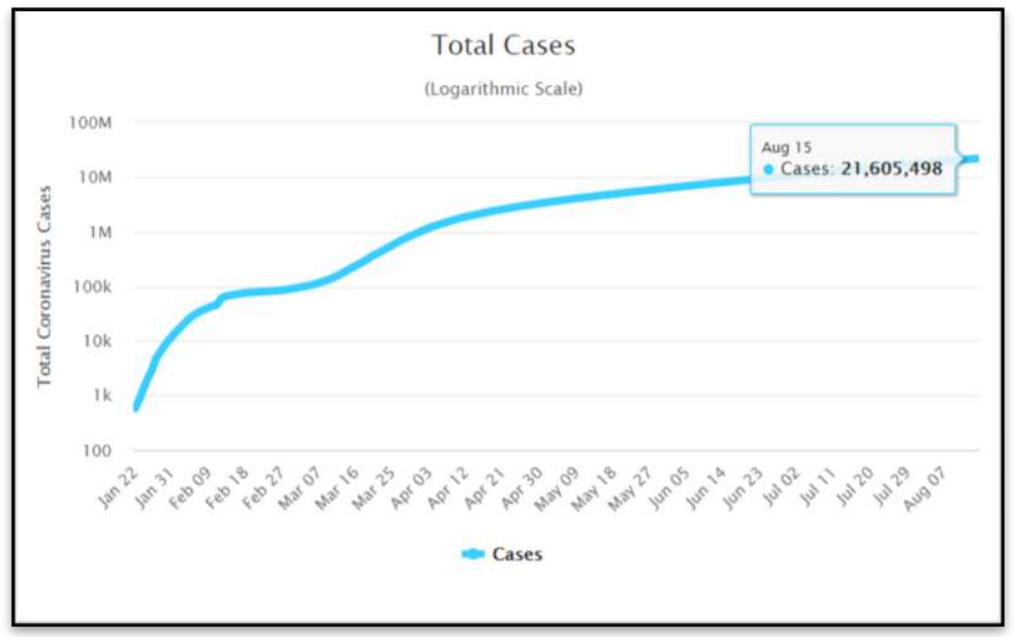

Fig 1(Corona Pandemic 2020 total Cases date-wise) 
Source -https://www.worldometers.info/coronavirus/worldwide-graphs/\#total-cases [accessed on 2020-08-16]

As with the present COVID-19 Pandemic situation, numbers of cases are rising day-by-day. If we compare the situation in last 6 month i.e. from January to mid of August 2020 we can clearly see that it is getting worse [Fig 1]. This situation arises because; we do not know how many people are carrying this virus, without getting noticed by the Government/ health personals. If there is any way to check that a person is getting the symptoms of a virulent disease at the place where the he is living or working, then it is easy to put him in isolation before the test of disease.

Further limitations and disadvantages of conventional and traditional approaches will become apparent to one of skill in the art through comparison of described systems with some aspects of the present disclosure, as set forth in the remainder of the present application and with reference to the drawings.

\subsection{Preliminaries}

Some of the key technologies used in this research are -

IoT: As there is advancement in sensing and networking technologies IoT (Internet of Things) become the most significant technology now-a-days. [11] We can describe IoT as the connection of peoples, sensors and services. It is used to decrease cost, improve efficiency, and increase the capability of existing system. [12]

CoT: Things (devices/sensors) of IoT when combines with the power of cloud, the new platform thus is termed as Cloud of Things (CoT). [13] In CoT cloud is in the middle of the architecture used as an interface between the different IoT devices communication each other. Cloud platform is also used to store and process the data generated via sensors used in IoT.

Fog Computing: Fog removes the problems associated with CoT, like performance and latency. It has the benefits of cloud, but removes demerits. [14] Fog gives storage, organization, distributed computing, and systems administration abilities closer to the client end. [15]

Block-Chain: Bitcoin has the foundation in Blockchain introduced by Satoshi Nakamoto. It can be defined as dispersed ledger, in which data and its transaction cannot be controlled by other then sender and receiver. [16] In the recent development other persons and companies developed their own blockchain. A main advantage of blockchain is to provide secured and encrypted way to process data and transaction.

Cloud Computing: Cloud provide way to share computing resources like server, storage, databases, network, software, etc in a scalable, and flexible environment. [16]. Cloud is a consumer involved technology; It is facilitated to end user as and when requested. [18] 


\subsection{Objective}

The main objectives of this research are -

1. To develop a system for predicting an infectious disease transmitted by a virulent respiratory virus. This system comprises -

a. Of a plurality of Internet of Things (IoT) sensors configured to be coupled to a plurality of human beings to generate a health dataset.

b. Of a plurality of fog node devices associated with a fog layer to receive the health dataset from the Internet of Things (IoT) sensors to process and to store the health dataset over a blockchain network, wherein the fog node devices process the health dataset at the fog layer by performing a fog computing

c. Of computing devices and cloud data centres to receive the processed health dataset from the fog node devices over the blockchain network.

2. To develop a method for predicting an infectious disease transmitted by a virulent respiratory virus, the method comprising steps of -

a. Generating health dataset from a plurality of Internet of Things (IoT) sensors configured to be coupled to a plurality of human beings.

b. transmitting, by the plurality of Internet of Things (IoT) sensors, the generated health dataset to a plurality of fog node devices associated with a fog layer for processing and storage of the health dataset over a blockchain network;

c. processing the health dataset at the fog layer by performing a fog computing; and

d. Transmitting, by the plurality of fog node devices, the processed health dataset to a plurality of computing devices and a plurality of cloud data centres over the blockchain network.

3. To develop a method according to point 1 , wherein the processed health dataset is transmitted to a software application associated with the computing devices accessible to a plurality of users. 


\section{Proposed Architecture}

\subsection{Architecture}

Fig 2 shows the proposed architecture of Blockchain \& FoT based VEPS (virus epidemic prediction system) Model. It is a model comprises of 4 layers.

1) Human layer: This layer contains the human beings from which the health data is gathered with the help of IoT sensors. There are number of wearable devices available for getting Health data like body temperature sensor, ECG sensor, blood pressure sensor, location sensor(for getting particular location of the person) etc. These sensors can be incorporated into a device like a wearable watch and then made available to all the people across the country.

2) Fog Layer: This layer is above the human layer and gets health data of humans routed via sensors and microcontrollers. This layer is at the city/area/district level where the data is being generated. The fog node devices are placed at a city level and at a district level. The fog node devices placed at the city level receive the generated health dataset from a city or a predefined area. The fog node devices placed at the district level receive the generated health dataset from a district. The fog node devices are associated with this layer to receive the health dataset from the plurality of Internet of Things (IoT) sensors to process and to store the health dataset over a blockchain network. The fog node devices process the health dataset at the fog layer by performing a fog computing.

3) Cloud Layer: The cloud data centers receive the processed health dataset from the fog node devices over the blockchain network. In our model Cloud layer is used to store processed data that can be made available countrywide/ worldwide.

4) Restricted Layer: The fog node devices placed at the district level are responsible for the processed data and transfer of that data between other nodes, government, and medical officials. The processed health dataset is transmitted to a software application associated with the computing devices accessible to a number of users such as government, health monitoring agencies, patient on the website or software application which is specific to users.

In this model we have used a blockchain network where processors provided with a memory configured to store a blockchain comprised of a number of blocks, wherein each block is comprised of at least a block header and the health dataset corresponding to the plurality of human beings, each block header including at least a timestamp. 


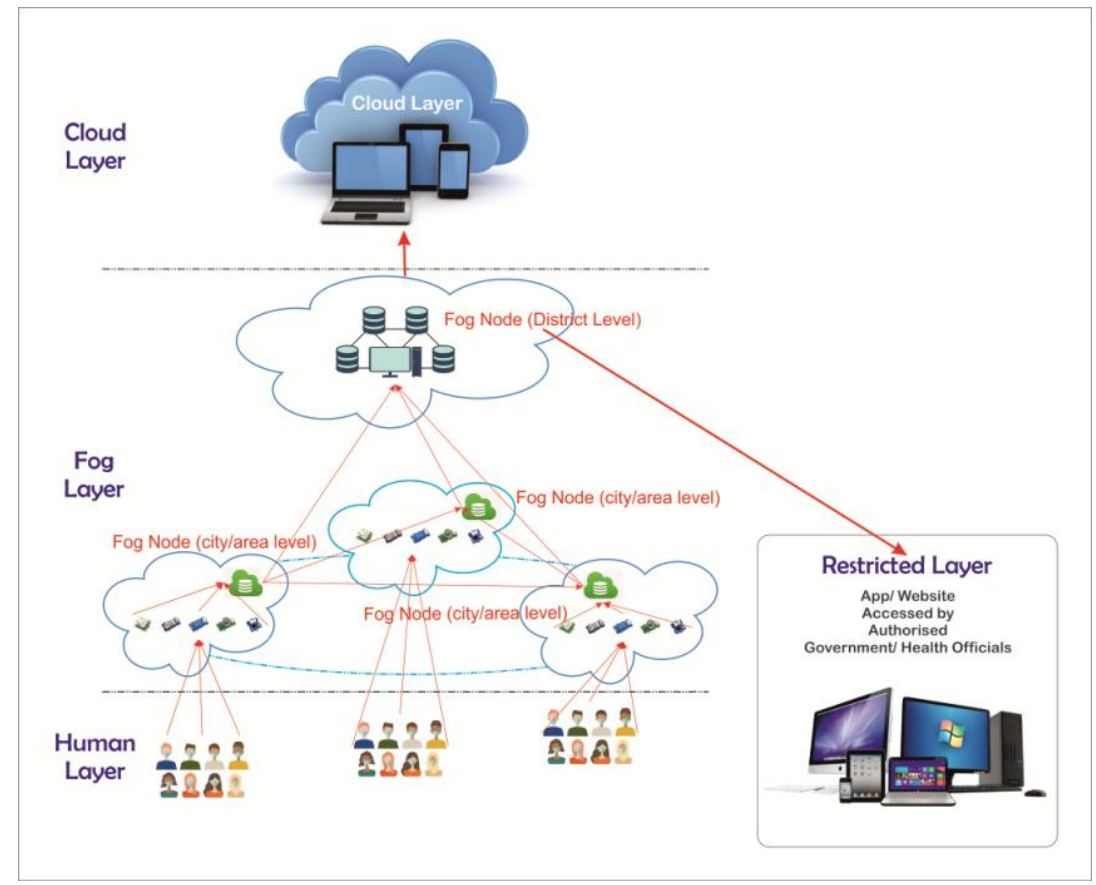

Fig 2 Architecture of VEPS

\subsection{Methodology - the Working flow of Proposed Architecture}

VEPS predicts the water-borne diseases and helps the consumer to improve water quality through WHO recommended standards.

1. The objective of the research is to get health data of human beings using secured blockchain \& FOT (Fog of Things).

2. The objective of the research is to identify health issues related to a viral Pandemic like COVID-19 in human beings.
a. High body Temperature
b. Increased Blood Pressure
c. ECG Sensor
d. Human Location sensor
e. etc

3. The health data will be transferred via IoT to the local Fog based System via secured blockchain technology.

4. Fog computing (Fog-of-Things) is used to perform processing of gathered data, before sending it to Cloud Data enters. 
5. The Fog System will automatically send alert to a mobile app/ computer Application accessible to all Government/ Health monitoring Agencies. This communication is also secured with blockchain.

6. A mobile app/Web Application connected to Fog Computing and Cloud data enters will be developed to-

a. Alert all officials of government and health monitoring agencies at the local district level about the suspected viral patient.

b. Trace the current location of the suspected patient.

c. To update the current status of Patient by Health officials after testing the patient (negative/ positive/ Quarantined/ Recovered/ Death etc).

d. Give information automatically of all Patients in a particular region/district/state.

7. All data from fog is transferred to the Cloud database via the blockchain network to use nationwide.

\subsection{Hardware \& Software (Technical) details included in Layers of Proposed Architecture}

1) Human Layer- Humans are provided with wearable sensors so that data is collected.

a. Some of the sensors that can be used are - ECG sensor, and a human location identification sensor (GPS sensor), temperature sensor (enabled for Raspberry Pi), heartbeat Sensor (enabled for Raspberry Pi),

2) Fog Layer- We can use different microcontroller boards available for getting data from IoT sensors like - Raspberry Pi, Particle.io, Espressif ESP8266 Boards, Adafruit, Arduino, etc.

a. In a single Fog Node we have routers, Networking interface, processing units, storage units etc. [7] so that it can communicate, store and process data.

b. Various application can be programmed for giving processing capabilities to this node using Python, Java etc. [7]

3) Cloud Layer- Different cloud databases are available. We can choose one from - Amazon Relational Database, Microsoft Azure SQL Database, Oracle Database, IBM Db2 on Cloud, Google Cloud SQL, etc.

4) Restricted Layer- In the restricted layer, an application is developed for giving further information to different user as described earlier. This application can be developed with the help of HTML/CSS \& PHP if it is web based or with Java/ Smalltalk/ Kotlin if it is app based. 


\subsection{System Flowchart}

Health dataset is generated with the help of loT sensors from humans

Health dataset generated thus transfered to Fog node device associated with fog layer over a blockchain network

Processing of health dataset at Fog Layer by performing Fog computing

Transmitting the processed Data to handheld Devices and Cloud Datacenters

\section{Mobile App/Website will then}

1) Alert all officials of government and health monitoring agencies at the local district level about the suspected viral patient.

2) Trace the current location of the suspected patient.

3) Update the current status of Patient by Health officials after testing the patient (negative/ positive/ Quarantined/ Recovered/ Death etc).

4) Give information automatically of all Patients in a particular region/district/state

\section{Evaluation}

\subsection{Advantage of proposed system VEPS}

Various advantages of the proposed system are -

1) This system automatically obtains a health dataset of human beings using a secured blockchain and FOT (Fog of Things).

2) It identifies various health issues such as high body temperature, and increased blood pressure, related to a viral epidemic like COVID-19 in human beings. 
3) It automatically provides information on all the patients in a particular region/district/state.

4) It prevents medical and governmental authorities from exposing directly to the infected patients as the system is equipped with intelligent IoT sensors such as an ECG sensor, a human location identification sensor etc.

We have incorporated a Block chain network in the system which has the following advantage -

1) It is decentralized; there is a minimal or absolutely no risk of data loss.

2) Blockchain relies heavily on cryptography (the practice of secure communication), so blockchain itself is a self-secure technology.

3) Using blockchain will also help the government authorities in surveillance, as the security authorities can look up for a particular health dataset of the human being, for the health dataset being decentralized. [17]

FoT (Fog of Things) brings the advantage to our system such as-

1) Fog removes the overload of computation and storage from the cloud platform.[9]

2) It is used to perform heavy computation on the edge where data gathers, that reduce the chance of data loss while sending it to the cloud.

3) Fog is also known to reduce latency period while communication.

4) FoT removes the resource sharing constraints of IoT devices.[10]

\section{Conclusion \& Future Scope}

In this study, we have developed a system named as VEPS for predicting an infectious disease such as COVID-19 transmitted by a virulent respiratory virus. This is a theoretical model and can be implemented as such on requirement. It includes technology as IoT, FoT, Cloud, Blockchain etc, and they have their own advantages. We have discussed these advantages in the paper.

This model is very helpful in the present epidemic situation of COVID-19. To stop spreading this virus early detection of patients is very much needed, so that they can be quarantined. Our prescribed system gives the opportunity to the health officials and government to get the location specific information about the person with health record.

Future work in this model will be to physical demonstration of this system. We can also try to figure out how the wearable devices can be made available peoples so that the health data can be gathered.

\section{References}

1. ImenBouzarkouna, Mohammed Sahnoun, NouhaSghaier, David Baudry, Christian Gout, "Challenges Facing the Industrial Implementation of Fog Computing", Future 
Internet of Things and Cloud (FiCloud) 2018 IEEE 6th International Conference, pp. 341-348, 2018.

2. C. Lee and A. Fumagalli, "Internet of Things Security - Multilayered Method For End to End Data Communications Over Cellular Networks," 2019 IEEE 5th World Forum on the Internet of Things (WF-IoT), Limerick, Ireland, 2019, pp. 24-28, doi: 10.1109/WF-IoT.2019.8767227.

3. S. Ziegler, "Considerations on IPv6 scalability for the Internet of Things — Towards an intergalactic Internet," 2017 Global Internet of Things Summit (GIoTS), Geneva, 2017, pp. 1-4, doi: 10.1109/GIOTS.2017.8016238.

4. Sanjay Sareen, Sunil Kumar Gupta \& Sandeep K. Sood (2017) An intelligent and secure system for predicting and preventing Zika virus outbreak using Fog computing, Enterprise Information Systems, 11:9, 1436-1456, DOI: 10.1080/17517575.2016.1277558

5. Dr. Naveen Tewari et al, "system and method for predicting an infectious disease transmitted by a virulent respiratory virus", Indian Patent Application No. 202011021969, Publication Date. 26/06/2020

6. Elmustafa Sayed Ali Ahmed, Mujtaba Elbagir Yousef, "Internet of things in Smart Environment: Concept, Applications, Challenges, and Future Directions", World Scientific News, WSN 134(1) (2019) 1-51, EISSN 2392-2192

7. Cha, H., Yang, H., \& Song, Y. (2018). A Study on the Design of Fog Computing Architecture Using Sensor Networks. Sensors, 18(11), 3633. doi:10.3390/s18113633

8. Mahmood, Z., \& Ramachandran, M. (2018). Fog Computing: Concepts, Principles and Related Paradigms. Fog Computing, 3-21. doi:10.1007/978-3-319-94890-4_1

9. N. Farjana, S. Roy, M. J. N. Mahi and M. Whaiduzzaman, "An identity-based encryption scheme for data security in fog computing", Proc. Int. Joint Conf. Comput. Intell., pp. 215-226, 2020.

10. A. A. Diro, N. Chilamkurti and Y. Nam, "Analysis of Lightweight Encryption Scheme for Fog-to-Things Communication," in IEEE Access, vol. 6, pp. 26820-26830, 2018, doi: 10.1109/ACCESS.2018.2822822.

11. K. L. Ang and J. K. P. Seng, "Application Specific Internet of Things (ASIoTs): Taxonomy, Applications, Use Case and Future Directions," in IEEE Access, vol. 7, pp. 56577-56590, 2019, doi: 10.1109/ACCESS.2019.2907793.

12. C. Perera, A. Zaslavsky, P. Christen and D. Georgakopoulos, "Context aware computing for the Internet of Things: A survey", IEEE Commun. Surveys Tuts., vol. 16, no. 1, pp. 414-454, 1st Quart. 2014.

13. M. M. E. Mahmoud et al., "Enabling Technologies on Cloud of Things for Smart Healthcare," in IEEE Access, vol. 6, pp. 31950-31967, 2018, doi: 10.1109/ACCESS.2018.2845399.

14. T. D. Dang and D. Hoang, "A data protection model for fog computing," 2017 Second International Conference on Fog and Mobile Edge Computing (FMEC), Valencia, 2017, pp. 32-38, doi: 10.1109/FMEC.2017.7946404.

15. M. Chiang, S. Ha, C.-L. I, F. Risso and T. Zhang, "Clarifying fog computing and networking: 10 questions and answers", IEEE Commun. Mag., vol. 55, no. 4, pp. 18-20, Apr. 2017.

16. W. Zheng, Z. Zheng, X. Chen, K. Dai, P. Li and R. Chen, "NutBaaS: A Blockchain-asa-Service Platform," in IEEE Access, vol. 7, pp. 134422-134433, 2019, doi: 10.1109/ACCESS.2019.2941905. 
17. H. Paik, X. Xu, H. M. N. D. Bandara, S. U. Lee and S. K. Lo, "Analysis of Data Management in Blockchain-Based Systems: From Architecture to Governance," in IEEE Access, vol. 7, pp. 186091-186107, 2019, doi: 10.1109/ACCESS.2019.2961404.

18. M. De Donno, K. Tange and N. Dragoni, "Foundations and Evolution of Modern Computing Paradigms: Cloud, IoT, Edge, and Fog," in IEEE Access, vol. 7, pp. 150936-150948, 2019, doi: 10.1109/ACCESS.2019.2947652.

19. David, C. et al (2004), "The severe acute respiratory syndrome coronavirus", Chiron Corporation, Patent No.-WO2004092360A2. World Intellectual Property Organization. 
Figures

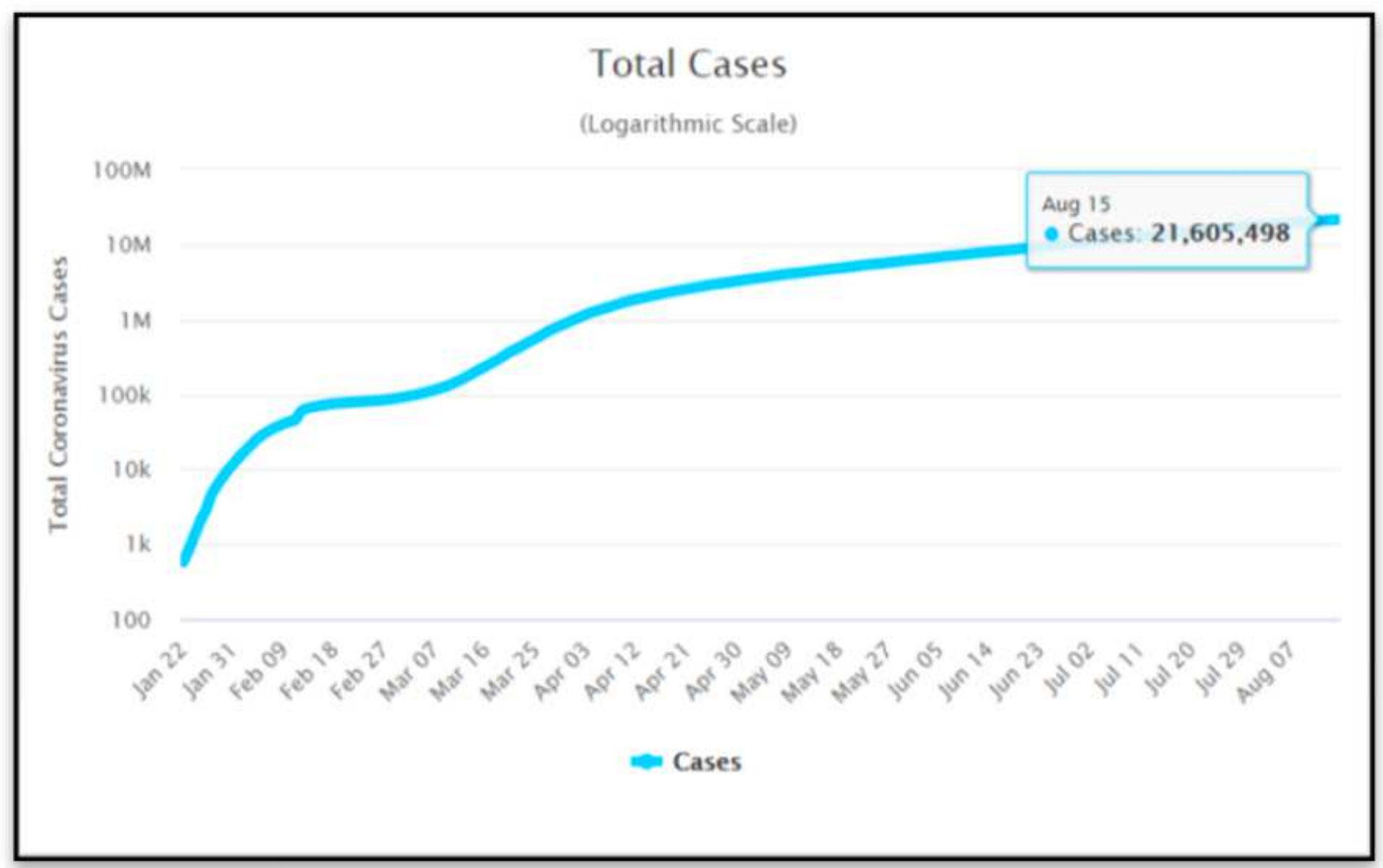

Figure 1

(Corona Pandemic 2020 total Cases date-wise) 


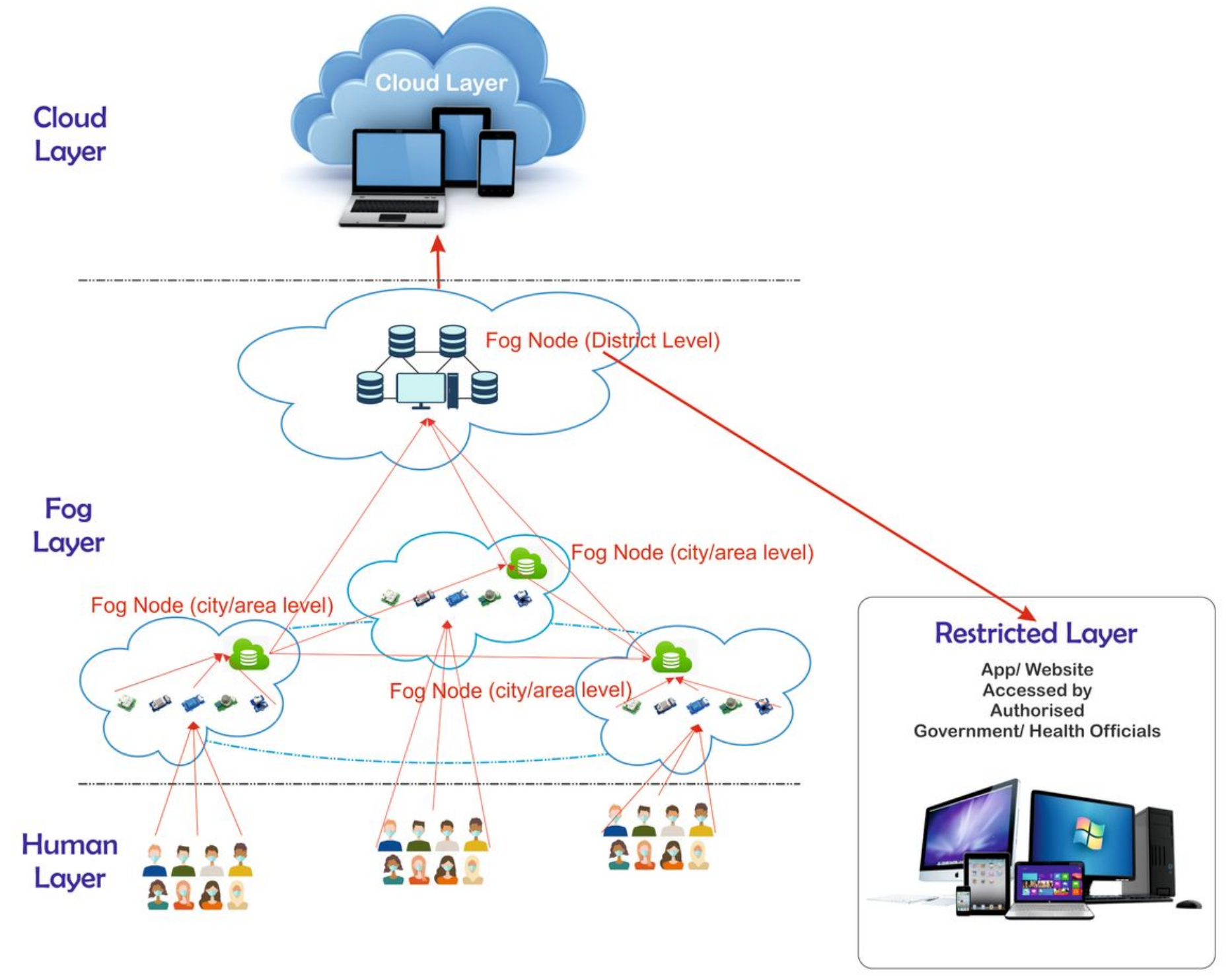

Figure 2

Architecture of VEPS 